\title{
Hypertensive Retinopathy and All-Cause Mortality in Older Adults of Amerindian Ancestry. A Population-based Longitudinal Prospective Study
}

\author{
Oscar H. Del Brutto ${ }^{1}$ (1) $\cdot$ Robertino Mera $^{2} \cdot$ Bettsy Y. Recalde $^{1} \cdot$ Denisse A. Rumbea ${ }^{1} \cdot$ Aldo F. Costa $^{3} \cdot$ Eduardo Viteri $^{4}$
}

Received: 23 August 2021 / Accepted: 21 October 2021 / Published online: 28 October 2021

(c) Italian Society of Hypertension 2021

\begin{abstract}
Introduction Hypertensive retinopathy (HTRP) predicts all-cause mortality in Asian and Caucasian populations. However, little is known about HTRP impact in other ethnic groups.

Aim We sought to estimate the mortality risk according to HTRP severity in older adults of Amerindian ancestry living in rural Ecuador.

Methods This prospective study enrolled individuals aged $\geq 60$ years with baseline blood pressure $\geq 120 / \geq 80 \mathrm{mmHg}$ from the ongoing Atahualpa Project cohort who received retinal photographs (for HTRP grading) and a brain MRI. We ascertained all-cause mortality after a mean of $5.2 \pm 1.2$ years of follow-up. Cox-proportional hazards models adjusted for demographics, cardiovascular risk factors, neuroimaging signatures of cerebral small vessel disease, blood pressure determinations during follow-up and incident strokes, were obtained to estimate mortality risk according to HTRP severity.

Results Analysis included 236 participants (mean age $69.3 \pm 7.3$ years). HTRP Grade 2 or higher was determined in 42 (18\%) individuals. Fifty participants (21\%) died during the follow-up, resulting in an overall unadjusted crude mortality rate of 4.1 per 100 person-years. Mortality rate in subjects with HTRP Grade 2 or higher was 7.2 and in those with no HTRP or Grade 1 only was 3.4 per 100 person-years. An adjusted Cox-proportional hazard model showed that individuals with HTRP Grade 2 or higher maintained a greater than two-fold mortality risk (HR 2.08; 95\% C.I. 1.04-4.15; $p=0.038$ ) when compared to those with no HTRP or Grade 1 only.

Conclusion Study results show that HTRP severity predicts mortality in this population of older adults.
\end{abstract}

Keywords Hypertensive retinopathy $\cdot$ Cerebral small vessel disease $\cdot$ Mortality $\cdot$ Prognosis $\cdot$ Population study

\section{Introduction}

Oscar H. Del Brutto

oscardelbrutto@hotmail.com

1 School of Medicine and Research Center, Universidad Espíritu Santo-Ecuador, Urbanización Toscana, Apt 3H, Km 4.5 vía Puntilla-Samborondón, 092301 Samborondón, Ecuador

2 Biostatistics/Epidemiology, Freenome, Inc., South San Francisco, CA, USA

3 Department of Neurology, Hospital Universitario Reina Sofía, Córdoba, Spain

4 Humana Visión Ophthalmological Center, Guayaquil, Ecuador
Arterial hypertension is a major public health problem affecting over 1 billion people worldwide [1]. Asymptomatic microvascular end-organ damage often precedes the development of more severe complications of hypertension such as cerebrovascular disease, acute myocardial infarction and death [2-4]. The retina is an early end-organ damage of arterial hypertension and, as such, the presence of hypertensive retinopathy (HTRP) may predict subsequent significant morbidity and increased mortality risk among affected individuals [5-7]. The goal would be early detection and implementation of intervention strategies aimed to reduce catastrophic consequences of arterial hypertension in subjects at risk. This can only be accomplished by means of a global preventive approach that should not be limited to prescription of antihypertensive medications [8]. 
While the increased risk of mortality in subjects with HTRP is well-known, some studies focused on patients with particular conditions attending specialized medical centers [9]. This information may not be generalized to the population of hypertensive individuals at large. Moreover, longitudinal population-based studies assessing mortality risk in patients with previously diagnosed HTRP have been conducted in Whites and Asian people living in developed countries [10-13], and these findings may not replicate the situation in remote rural settings, where living conditions and access to medical care are completely different. In addition, more information is needed to assess possible racial/ ethnic disparities in the risk of mortality associated with HTRP, as has been suggested in the prevalence of this condition across African-Americans and Whites [14].

Taking the opportunity of the well-established Atahualpa Project Cohort, our group previously conducted a population-based study aimed to assess the association between HTRP and the presence of neuroimaging signatures of cerebral small vessel disease (cSVD), as proof of the simultaneous and paucisymptomatic damage of two different endorgans of arterial hypertension (the retina and the brain) [15]. In order to expand our understanding on the relationship between HTRP and mortality risk, those individuals were enrolled in a prospective longitudinal study aimed to assess whether HTRP predicts all-cause mortality in community-dwelling older adults living in a remote rural setting.

\section{Methods}

\subsection{Study Population}

Subjects were participants of the Atahualpa Project, a population-based cohort focused on risk factors that are increasing the burden of cardiovascular and neurological diseases in rural coastal Ecuador [16]. Individuals with a baseline arterial pressure $\geq 120 / \geq 80 \mathrm{mmHg}$ who received a brain MRI (from 2013 to 2015) and retinal photographs (from September to November 2015) were eligible to participate in this study [15], and those who signed a comprehensive informed consent agreeing to be included in this prospective study were enrolled. Characteristics of the village and its residents have been detailed elsewhere [17]. In brief, Atahualpa is an isolated community, and residents are homogeneous regarding race/ethnicity (Amerindian ancestry), socioeconomic status and dietary habits. The migration rate is low and participant retention in the project is high, which makes Atahualpa an optimal setting for the longitudinal followup of cohort studies [18]. The study was approved by the Institutional Review Board of Hospital-Clínica Kennedy, Guayaquil.

\subsection{Retinal Photographs}

Retinal photographs were taken with the use of the Easy Scan v1.2 (i-Optics B.V., The Hague, The Netherlands), as previously detailed [15]. This zero-dilatation smart retinal imaging system uses confocal line scanning laser ophthalmoscope technology to illuminate the retina through a $2 \mathrm{~mm}$ pupil, and can better penetrate media opacities than conventional fundus cameras. All exams were performed at the headquarters of the Atahualpa Project by trained medical students. Then, a certified ophthalmologist with more than 30 years of experience reviewed all images blinded to clinical data and MRI findings. The Keith-Wagener-Barker classification was used for HTRP grading [19]. This includes: Grade 1, mild generalized retinal arteriolar narrowing; Grade 2, definite focal narrowing and arteriovenous nicking; Grade 3, signs of grade 2 retinopathy plus retinal hemorrhage, exudate, and cotton-wool spots; and Grade 4, signs of grade 3 retinopathy plus papilledema. When HTRP grades were different between the two eyes, the more advanced grade was used for analyses.

\subsection{Neuroimaging Studies}

Brain MRIs were performed with a Philips Intera 1.5T (Philips Medical Systems, Eindhoven, the Netherlands), following proposed standards for research on cSVD [20]. MRI readings focused on white matter hyperintensities (WMH) of presumed vascular, deep cerebral microbleeds (CMB), lacunes of presumed vascular origin, and enlarged basal ganglia perivascular spaces (BG-PVS). WMH were defined as hyperintense lesions on T2-weighted images that remained bright on FLAIR (without cavitation) and graded according to the modified Fazekas scale [21]. WMH were considered moderate-to-severe when periventricular lesions extended into the deep white matter or deep lesions became confluent or semi-confluent. CMB were identified as small $(\leq 10 \mathrm{~mm})$ round areas of signal void with blooming seen on gradient-echo sequences and rated according to the microbleed anatomical rating scale [22]. Lacunes were defined as fluid-filled cavities measuring 3-15 $\mathrm{mm}$ in diameter located in the territory of perforating arterioles [20]. Enlarged BGPVS were defined as small $(<3 \mathrm{~mm})$ sharply-delineated spaces of CSF intensity on T2-weighted images that followed the course of perforating arterioles. Enlarged BG-PVS were graded at the level of the basal ganglia and considered moderate-to-severe if $>10$ lesions were seen in one side of the brain [23]. In addition, non-lacunar strokes were identified and classified according to their specific stroke subtype.

MRIs were independently read by two investigators blinded to clinical data. Kappa coefficients for inter-reader 
agreement were 0.91 for moderate-to-severe $\mathrm{WMH}, 0.82$ and 0.90 for $\mathrm{CMB}$ and lacunes presence, respectively, 0.84 for enlarged BG-PVS, and 0.92 for non-lacunar strokes; disagreements were resolved by consensus

\subsection{Outcome Measures}

Study participants were screened yearly to determine changes in vital status. Ambulatory blood pressure was determined at every visit according to a previously described protocol [17]. Likewise, a validated field instrument designed to determine new (incident) strokes was applied to all individuals and suspected cases were examined by a certified neurologist—blinded to the results of fundoscopic examination - with the aid of information provided by MRI. In addition, field personnel continuously visited households were participants live and reminded them and their proxies to notify us in case of a new major health problem or in the event of death. Positive non-fatal outcomes were evaluated by participants' or proxy-delivered medical history and medical records review (in case of admission to neighboring hospitals); then, a certified neurologist confirmed the diagnosis of incident stroke as previously described. Death certificates were reviewed and verbal autopsies were obtained to ascertain the date and probable cause of death. For the current study, the last censoring date was set as the 1st of June 2021. Participants who declined consent and those who emigrated were censored at the last annual survey when the individuals were interviewed, and those who died were censored at the time of death.

\subsection{Clinical Covariates}

Demographics (age, gender, level of education), cardiovascular risk factors, the mean of several systolic and diastolic blood pressure determinations during the study years, and development of a new-onset stroke during the follow-up were selected as covariates. Age was recorded at the time of retinal photographs. Cardiovascular risk factors were assessed at baseline by means of the American Heart Association (AHA) criteria, and determined as poor as follows: current smoker status, a body mass index $\geq 30 \mathrm{~kg} / \mathrm{m}^{2}$, no moderate and vigorous physical activity, intake of $0-1$ components of the healthy diet proposed by the AHA, fasting glucose $\geq 126 \mathrm{mg} / \mathrm{dL}$, and total cholesterol levels $\geq 240 \mathrm{mg} /$ dL [24]. Baseline blood pressure, also included by the AHA as a cardiovascular risk factor, was not used as a covariate because of collinearity.

\subsection{Statistical Analysis}

All data analyses were carried out by using STATA version 17 (College Station, TX, USA). Baseline characteristics of the study population were compared according to HTRP severity (dichotomized in none or grade 1, and grade 2 or higher) using linear models for continuous variables and the chi-squared test or the Fisher exact test for categorical variables. We calculated the crude mortality incidence rate ratio (IRR) overall and stratified by HTRP severity using Poisson regression. To compute the person-years of follow-up we considered the time from retinal photographs to the last censoring date, study drop-out, or time of death. A Coxproportional hazard model (Breslow method for ties) was fitted to calculate the Hazard Ratio (HR) with its 95\% confidence interval (CI) in order to estimate the risk for all-cause mortality according to the presence of HTRP Grade 2 or higher, after adjusting for the above-mentioned clinical and neuroimaging covariates.

\section{Results}

Of 241 individuals receiving baseline retinal photographs and brain MRIs, five declined consent for the present cohort study and were not included. HTRP Grade 2 or higher was determined in 42 of 236 (18\%) individuals.

The mean age (at baseline) of study participants was 69.3 \pm 7.3 years (median 67.8 years), $138(58 \%)$ were women, and $191(81 \%)$ had primary school education only. Cardiovascular risks factors were distributed as follow: six (3\%) were current smokers, 47 (20\%) were obese, 16 (7\%) were physically inactive, $10(4 \%)$ had an unhealthy diet, 79 (33\%) had hyperglycemia, and 31 (13\%) had hypercholesterolemia. Moderate-to-severe WMH were detected in 49 (21\%) participants, CMB in $24(10 \%)$, lacunes in $31(13 \%)$ and $>10$ enlarged BG-PVS in 69 (29\%). A total of 18 (8\%) study participants had baseline non-lacunar strokes. The mean systolic and diastolic blood pressure determinations during follow-up were $135.4 \pm 16.9$ and $73.3 \pm 9.7 \mathrm{mmHg}$, respectively, and 17 (7\%) individuals developed an incident stroke. During follow-up, five participants abandoned the study (emigration).

Fifty participants (21\%) died during a mean follow-up of $5.2 \pm 1.2$ years (range $0.6-5.8$ ), median: 5.7 years, IQR 5.6-5.8 (total years of follow-up 1236), resulting in an overall unadjusted crude mortality rate of 4.1 (95\% C.I. 2.9-5.2) per 100 person-years of follow-up. Mortality rate in subjects with HTRP Grade 2 or higher was 7.2 (95\% C.I. 3.5-10.8) and in those no HTRP or Grade 1 only was 3.4 (95\% C.I. 2.3-4.5) per 100 person-years of follow-up.

Table 1 summarizes participants' characteristics according to HTRP severity. In unadjusted analysis, having moderate-to-severe WMH, > 10 enlarged BG-PVS, and non-lacunar strokes were significantly associated with HTRP Grade 2 or higher, while being obese was inversely associated with HTRP Grade 2 or higher. In addition, mortality was 
Table 1 Characteristics of Atahualpa residents aged $\geq 60$ years according to the presence and severity of hypertensive retinopathy (univariate analyses)

\begin{tabular}{|c|c|c|c|c|}
\hline & Total series $(n=236)$ & $\begin{array}{l}\text { Normal retina and HTRP } \\
\text { Grade } 1(n=194)\end{array}$ & $\begin{array}{l}\text { HTRP } \\
\text { Grade } 2 \text { or higher }(n \\
=42)\end{array}$ & $p$ value \\
\hline Age, years $($ mean $\pm \mathrm{SD})$ & $69.3 \pm 7.3$ & $69.5 \pm 7.5$ & $68.4 \pm 5.9$ & 0.373 \\
\hline Women, $n(\%)$ & $138(58)$ & $112(58)$ & $26(62)$ & 0.619 \\
\hline Primary school education, $n(\%)$ & $191(81)$ & $158(81)$ & $33(79)$ & 0.668 \\
\hline Current smoker, $n(\%)$ & $6(3)$ & $4(2)$ & $2(5)$ & 0.290 \\
\hline Body mass index $\geq 30 \mathrm{~kg} / \mathrm{m}^{2}, n(\%)$ & $47(20)$ & $42(22)$ & $5(12)$ & $<0.001 *$ \\
\hline Poor physical activity, $n(\%)$ & $16(7)$ & $12(6)$ & $4(10)$ & 0.496 \\
\hline Poor diet, $n(\%)$ & $10(4)$ & $9(5)$ & $1(2)$ & 0.510 \\
\hline Fasting glucose $\geq 126 \mathrm{mg} / \mathrm{dL}, n(\%)$ & $79(33)$ & $61(31)$ & $18(43)$ & 0.155 \\
\hline Total cholesterol $\geq 240 \mathrm{mg} / \mathrm{dL}, n(\%)$ & $31(13)$ & $23(12)$ & $8(19)$ & 0.211 \\
\hline Moderate-to-severe WMH, $n(\%)$ & $49(21)$ & $34(18)$ & $15(36)$ & $0.008^{*}$ \\
\hline Cerebral microbleeds, $n(\%)$ & $24(10)$ & $18(9)$ & $6(14)$ & 0.330 \\
\hline Lacunes, $n(\%)$ & $31(13)$ & $22(11)$ & $9(21)$ & 0.079 \\
\hline$>10$ enlarged BG-PVS, $n(\%)$ & $69(29)$ & $47(24)$ & $22(52)$ & $<0.001$ \\
\hline Non-lacunar strokes, $n(\%)$ & $18(8)$ & $11(6)$ & $7(17)$ & $0.015^{*}$ \\
\hline Systolic pressure (follow-up), mean \pm SD & $135.4 \pm 16.9$ & $135.3 \pm 16.6$ & $136.2 \pm 18.4$ & 0.755 \\
\hline Diastolic pressure (follow-up), mean $\pm \mathrm{SD}$ & $73.3 \pm 9.7$ & $72.9 \pm 9.6$ & $75.4 \pm 9.9$ & 0.129 \\
\hline Incident stroke, $n(\%)$ & $17(7)$ & $14(7)$ & $3(7)$ & 0.987 \\
\hline Mortality, $n(\%)$ & $50(21)$ & $35(18)$ & $15(36)$ & $0.011^{*}$ \\
\hline
\end{tabular}

$H T R P$ hypertensive retinopathy, $B G-P V S$ enlarged basal ganglia-perivascular spaces

*Statistically significant result

significantly associated with HTRP Grade 2 or higher [15/42 (36\%) versus 35/194 (18\%), $p=0.011]$. The probable cause of death vas vascular (stroke, acute myocardial infarction) in 13 individuals and non-vascular (mostly end-stage kidney disease, liver cirrhosis, or cancer) in 25 . The remaining 12 subjects died during the acute phase of severe COVID-19. There were no significant differences in the probable cause of death across grades of HTRP severity; however, small numbers preclude proper analysis of this data.

A Cox-proportional hazard model showed that individuals with HTRP Grade 2 or higher maintained a greater than two-fold mortality risk (HR 2.08; 95\% C.I. $1.04-4.15 ; p=$ 0.038 ) when compared to those with no HTRP or Grade 1 only, after adjusting for covariates of interest (Fig. 1). In this
Fig. 1 Kaplan-Meier survival curves and Hazard Ratios with 95\% confidence intervals for all-cause mortality according to hypertensive retinopathy severity, adjusted for demographics and cardiovascular risk

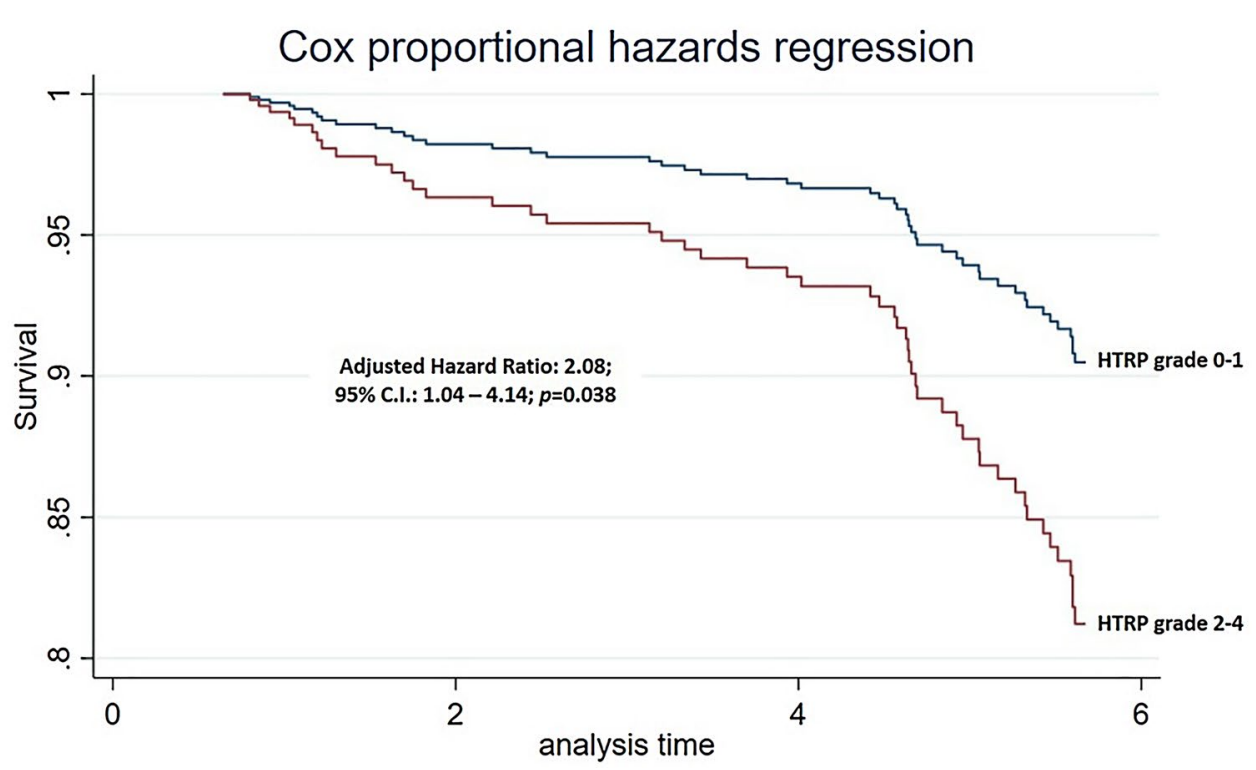


model, several covariates reached independent significance in the relationship between HTRP severity and mortality, including: increasing age $(p<0.001)$, primary school education only $(p=0.044)$, unhealthy diet $(p=0.028)$, presence of lacunes $(p=0.019)$, and the mean values of systolic and diastolic blood pressures during follow-up $(p<0.001)$. The reason why age remained as a significant covariate in the Cox-proportional hazard model but not in univariate analysis (Table 1) is because increasing age is associated with mortality, not with HTRP severity.

A multivariate Poisson regression model compared mortality rates using ordinal categories of HTRP as independent variables. In this model—using the category of "no HTRP" as the reference value-there was no difference in the IRR of mortality when compared to individuals having HTRP Grade 1 (IRR 1.13; 95\% C.I. 0.58-2.19; $p=0.723$ ), but there was an increased IRR when compared with those with HTRP Grade 2 (IRR 2.13; 95\% C.I. 1.04-4.39; $p=0.040$ ) and Grades 3-4 (IRR 4.72; 95\% C.I. 1.38-16.1; $p=0.013$ ). The above-mentioned model took into account the months of follow-up.

\section{Discussion}

In this population-based cohort of pre-hypertensive and hypertensive older adults living in a rural setting, HTRP severity predicts all-cause mortality independently of demographics, cardiovascular risk factors, neuroimaging signatures of cSVD, non-lacunar stroke subtypes, incident strokes and blood pressure control during follow-up.

As previously mentioned, only few population-based studies have addressed mortality risk among individuals with HTRP [10-13]. In the Beaver Dam Eye Study, the authors reviewed baseline retinal photographs of 413 individuals who died from cardiovascular diseases over a 10-year period (case-patients) and compared the prevalence of retinopathy — not necessarily HTRP — in this group with that of 1,198 study participants (controls) who remained alive during those years. A total of $80(20.3 \%)$ individuals who died and 126 (11\%) of those who remained alive had retinopathy, for an adjusted odd ratio of 1.8 (95\% C.I. 1.2-2.7) [10]. Another study in the same population-confined to non-diabetic individuals-retinopathy was associated with a higher risk of all-cause and cerebrovascular mortality (HR 1.76; 95\% C.I. 1.16-2.69, and HR: 3.17; 95\% C.I. $1.73-5.78$, respectively) after 14 years of follow-up; the total sample of this study was 4294 individuals (3059 survivors and 1,235 deaths) [11].

In the largest study conducted to date, 87,890 Japanese individuals aged 40-79 years, with arterial hypertension or not, were followed-up for a mean of 14 years. Those with grades 3-4 HTRP at baseline were not taken into account.
The multivariable hazard ratios for all-cause mortality and cardiovascular disease mortality were higher in individuals with HTRP grade 2 when compared with those with no HTRP or HTRP grade 1 ( $p$ for trend < 0.01) [12]. Another study, using 7640 participants of the National Health and Nutrition Examination Survey (NHANES), disclosed that the risk for all-cause mortality was significantly higher in subjects presenting with HTRP plus chronic kidney disease than in those with HTRP alone (HR 239; 95\% C.I. 1.77-3.22 versus 1.02 ; 95\% C.I. 0.75-1.38) [13].

While, in general terms, the above-mentioned studies are in line with results of the present study, study designs and procedures are not totally comparable. For example, none of these studies systematically performed brain MRIs at baseline in all study participants. Therefore, pre-existing cerebrovascular damage of study participants could not be accurately determined in view of the several asymptomatic lesions that may subsequently result in the development of catastrophic consequences such a stroke-related death.

A debated question is whether aggressive treatment of blood pressure levels below than standard targets is beneficial in reducing mortality in subjects with HTRP without taking into account the complete set of cardiovascular risk factors or pre-existing asymptomatic or paucisymptomatic cerebrovascular diseases [8]. It is possible that other lifestyle interventions such as improving dietary habits, weight loss, strict glucose control, and smoking cessation may provide additional benefit in individuals with HTRP. In our series, for example, an unhealthy diet remaining as a significantly independent covariate in the model assessing the relationship between HTRP severity and all-cause mortality. More information is needed for the rational implementation of cost-effective intervention strategies among these individuals.

The present study has several strengths that include the population-based design, the assessment of retinal photographs by means of a uniform and standardized protocol, the systematic practice of neuroimaging studies (MRIs) that focused on the presence of baseline cerebrovascular lesions, and the detailed clinical characterization and follow-up of the study population. Study limitations include low generalizability to other populations, absence of cause of death sub-analysis due to suspected inaccuracies in death certificates and verbal autopsy reports and limited number of fatal outcomes, and the lack of investigation of other hypertension-related end-organ damage (kidneys and the heart) that may have contributed to the higher mortality rate among individuals with HTRP. In addition, it is possible that some unmeasured covariates accounted for part of the disclosed relationship between HTRP and mortality.

In this population-based study, participants were taken from the community and not from specialized clinics. Therefore, the frequency of individuals with HTRP grades 
3-4 was low (only 4 subjects) and three of them died in the follow-up. While these numbers may be too low to get valid conclusions, the Poisson regression model showed a linear effect on mortality when different HTRP Grades were individually compared, suggesting an independent increased risk of mortality when subjects with HTRP Grades 2 or higher when compared to those of no HTRP.

In summary, HTRP Grade 2 or higher is associated with all-cause mortality in community-dwelling Amerindians living in a rural setting, similar to that previously reported in other races/ethnic groups living in urban centers. Incidental diagnosis of HTRP is a reliable and practical tool to predict adverse outcomes, even in remote populations with limited access to healthcare. Despite the absence of high-quality evidence, interventions aimed to a complete cardiovascular risk control may result cost-effective in underserved populations to reduce adverse outcomes related to HTRP.

Funding This study was partially supported by Universidad Espíritu Santo-Ecuador, Guayaquil, Ecuador.

\section{Declarations}

Conflict of Interest All authors report no conflicts of interest to disclose.

Ethical Approval The study was approved by the Institutional Review Board of Hospital-Clínica Kennedy, Guayaquil, Ecuador (FWA 00030727). All subjects signed an informed consent form before enrollment.

Informed Consent All subjects signed an informed consent form before enrollment.

\section{References}

1. http://www.emro.who.int/media/world-health-day/public-healthproblem-factsheet-2013.html.

2. Piskorz D. Hypertensive mediated organ damage and hypertension management. How to assess beneficial effects of antihypertensive treatments? High Blood Press Cardiovasc Prev. 2020; 27: 9-17.

3. Shlomai G, Grassi G, Grossman E, Mancia G. Assessment of target organ damage in the evaluation and follow-up of hypertensive patients: Where do we stand? J Clin Hypertens (Greenwich). 2013;15:742-7.

4. Wong TY, Klein R, Couper DJ, Cooper LS, Shahar E, Hubbard LD, et al, Sharrett AR. Retinal microvascular abnormalities and incident stroke: the Atherosclerosis Risk in Communities Study. Lancet. 2001; 358: 1134-40.

5. Wong TY, McIntosh R. Hypertensive retinopathy signs as risk indicators of cardiovascular morbidity and mortality. Br Med Bull. 2005;73-74:57-70.

6. Hanff TC, Sharrett AR, Mosley TH, Shibata D, Knopman DS, Klein $\mathrm{R}$, et al. Retinal microvascular abnormalities predict progression of brain microvascular disease. An Atherosclerotic Risk in Communities Magnetic Resonance Imaging Study. Stroke. 2014; 45:2012.7.

7. Moss HE. Retinal vascular changes are a marker for cerebral vascular diseases. Curr Neurol Neurosci Rep. 2015;15:40.
8. Le Bao Ho C, Breslin M, Doust J, Reid CM, Nelson MR. Effectiveness of blood pressure-lowering drug treatment by levels of absolute risk: post-hoc analysis of the Australian National Blood Pressure Study. BMJ Open. 2018; 8: e017723.

9. Jibran MS, Habib SA, Khan SB. Hypertensive retinopathy. A prognostic factor for morbidity and mortality after acute ST elevation myocardial infarction. J Coll Physicians Surg Pak 2019; 29: 205-9.

10. Wong TY, Klein R, Nieto FJ, Klein BEK, Sharrett AR, Meuer SM, et al. Retinal microvascular abnormalities and 10-year cardiovascular mortality. A population-based case-control study. Ophthalmology. 2003;110:933-40.

11. Hirai FE, Moss SE, Knudtson MD, Klein BEK, Klein R. Retinopathy and survival in a population without diabetes. The Beaver Dam Eye Study. Am J Epidemiol. 2007; 166: 724-30.

12. Sairenchi T, Iso H, Yamagishi K, Irie F, Okubo Y, Gunji J, et al. Mild retinopathy is a risk factor for cardiovascular mortality in Japanese with an without hypertension. The Ibaraki prefectural health study. Circulation. 2011;124:2502-11.

13. Ricardo AC, Grunwald JE, Parvathaneni S, Goodin S, Ching A, Lash JP. Retinopathy and CKD as predictors of all-cause and cardiovascular mortality: national health and nutrition examination survey (NHANES) 1988-1994. Am J Kidney Dis. 2014;64:198-203.

14. Wong TY, Klein R, Duncan BB, Nieto FJ, Klein BEK, Couper DJ, et al. Racial differences in the prevalence of hypertensive retinopathy. Hypertension. 2003;41:1086-91.

15. Del Brutto OH, Mera RM, Viteri EM, Pólit J, Ledesma EA, Cano JA, et al. Hypertensive retinopathy and cerebral small vessel disease in Amerindians living in rural Ecuador: The Atahualpa Project.

16. Del Brutto OH, Mera RM, Castillo PR, Del Brutto VJ. Key findings from the Atahualpa project: What should we learn? Expert Rev Neurother. 2018;18:5-8.

17. Del Brutto OH, Peñaherrera E, Ochoa E, Santamaría M, Zambrano M, Del Brutto VJ. Door-to-door survey of cardiovascular health, stroke, and ischemic heart disease in rural coastal Ecuador-the Atahualpa Project: methodology and operational definitions. Int J Stroke. 2014;9:367-71.

18. Del Brutto OH, Castillo PR, Sedler MJ, Del Brutto VJ, Zambrano M, Mera RM, et al. Reasons for declining consent in a populationbased cohort study conducted in a rural South American community. J Environ Public Health. 2018;2018:8267948.

19. Downie LE, Hodgson LAB, D'Sylvia C, McIntosh RL, Rogers SL, Connell $\mathrm{P}$, et al. Hypertensive retinopathy: comparing the KeithWagener-Barker to a simplified classification. J Hypertension. 2013;31:960-5.

20. Wardlaw JM, Smith EE, Biessels GJ, Cordonnier C, Fazekas F, Frayne R, et al. Neuroimaging standards for research into small vessel disease and its contribution to ageing and neurodegeneration. Lancet Neurol. 2013;12:822-38.

21. Fazekas F, Chawluk JB, Alavi A, Hurtig HI, Zimmerman RA. MR signal abnormalities at $1.5 \mathrm{~T}$ in Alzheimer's dementia and normal aging. AJR Am J Roentgenol. 1987; 149: 351-6.

22. Gregoire SM, Chaudhary UJ, Brown MM, Yousry TA, Kallis C, Jager HR, et al. The Microbleed Anatomical Rating Scale (MARS): reliability of a tool to map brain microbleeds. Neurology. 2009;73:1759-66.

23. Doubal FN, MacLullich AM, Ferguson KJ, Dennis MS, Wardlaw JM. Enlarged perivascular spaces on MRI are a feature of cerebral small vessel disease. Stroke. 2010;41:450-4.

24. Lloyd-Jones D, Hong Y, Labarthe D, Mozaffarian D, Appel LJ, Van Horn L, et al. Defining and setting national goals for cardiovascular health promotion: the American heart association's strategic impact goal through 2020 and beyond. Circulation. 2010;121:586-613. 